\title{
Privatisierung und Vermarktlichung der Altersvorsorge: Eingetrübte Aussichten des deutschen Mehrsäulenmodells
}

\author{
Bernhard Ebbinghaus
}

WSI-Mitteilungen, 06/2018 (https://www.boeckler.de/wsi-mitteilungen_117218 117235.htm)

Die deutsche lebensstandardsichernde Rente, finanziert aus Sozialabgaben der Versicherten und ihrer Arbeitgeber, galt jahrzehntelang als Blaupause des Bismarck'schen Modells. Seit 20 Jahren, unter dem demografischen Druck und den fiskalischen Restriktionen, wurde der umfassende Umbau in ein Mehrsäulensystem betrieben. Die kapitalgedeckte Riester-Rente hatte einen schwierigen Start, und die Erfahrung der jüngsten Finanzmarktkrisen verdeutlicht grundsätzliche Probleme der kapitalgedeckten Altersvorsorge. Auch die betriebliche Altersvorsorge benötigt tarifpolitische Weiterentwicklung. Die derzeitigen Formen der Alterssicherung können die Risiken steigender Altersarmut in einer flexibleren Arbeitsgesellschaft nur ungenügend auffangen, und es bedarf allemal einer besseren Mindestsicherung für alle Mitbürger.

\section{Alterssicherung im Wandel}

Die Sicherung des Einkommens im Alter und bei Erwerbsminderung ist eine wesentliche Errungenschaft des deutschen Sozialstaates. Noch vor 70 Jahren, während der unmittelbaren Nachkriegszeit, galten Altersarmut unter Kriegswitwen, der Verlust des Ersparten und kriegsbedingte Erwerbsunfähigkeit als drängende gesellschaftliche Probleme. Als Teil der sozialen Marktwirtschaft schuf die Rentenreform von 1957 den „Generationenvertrag“ zwischen Rentenbeziehern und Beitragszahlern, zwischen gegenwärtigen und zukünftigen Rentnergenerationen. Als Bismarck'sches Paradebeispiel einer Sozialversicherung ermöglichte die deutsche gesetzliche Rentenversicherung (GRV) dank Umlageverfahren für mehre Jahrzehnte die Sicherung des Lebensstandards im Alter. Während viele kontinentaleuropäische Nachbarländer diesem Pfad folgten, schufen die Briten mit den Beveridge-Reformen bereits ab 1946 ein Mehrsäulenmodell aus universeller Grundrente und privater Zusatzversorgung; diesem Weg folgten auch die Iren, Niederländer, Schweizer und Skandinavier (Meyer 2013). Finanzmarktkapitalismus trat in diesen Mehrsäulenmodellen viel früher in Erscheinung als in Deutschland. Nach dem Mauerfall führten viele Länder Osteuropas kapitalgedeckte Zusatzversorgungen als Liberalisierungspolitik und Forderung ihres Finanzmarktes ein. Auch Deutschland schlug den Weg der Vermarktlichung und Privatisierung der Alterssicherung nach der deutschen Einigung ein.

Deutschland steht eher am Beginn einer fundamentalen Umorientierung der Altersvorsorge, auch wenn bereits seit drei Jahrzehnten die Alterung der deutschen Gesellschaft als Herausforderung für eine nachhaltige Alters und Rentenpolitik gilt. Am Abend des Mauerfalls wurde die erste umfassendere Rentenreform verabschiedet, die die ersten Schritte zu einer Neuarchitektur der Alterssicherung ab 1992 einleitete. Sie wagte den langsamen Ausstieg aus der Frühverrentung, die seit den Ölkrisen der 1970er Jahre zur Entlastung und 
Restrukturierung des Arbeitsmarktes betrieben wurde. Weitere Reformen um die Jahrtausendwende haben die Rentenpolitik im geeinten Deutschland umfassend neubestimmt : Neben der staatlichen Abstützung sollen die bereits vorhandene betriebliche und eine neue individuelle Altersvorsorge weitere Stutzpfeiler bieten, um den bisherigen Lebensstandard im Alter zu halten, da dieser wegen des demografischen Wandels nicht mehr alleine durch den staatlichen Pfeiler einer Sozialversicherung abgesichert werden kann.

Deutschland steht nicht alleine beim Aufbau einer Mehrsäulenarchitektur der Alterssicherung in Europa (469) (Meyer 2015). Die deutschen Reformen zu Beginn des Millenniums zeigen exemplarisch, wie Vermarktlichung und Privatisierung gemeinsam betrieben wurden, um einerseits eine Stärkung des Finanzmarktes voranzutreiben und eine Entlastung staatlicher Ausgaben zu erzielen. Vermarktlichung beinhaltet nicht nur den Ausbau kapitalgedeckter Altersvorsorge, sondern auch die Stärkung des Beitragsprinzips und demografische Nachhaltigkeitsanpassungen im staatlichen Umlagesystem. Die langfristige Kürzung der staatlichen Renten führt zu einer Versorgungslücke der Lebensstandardsicherung, die den Handlungsdruck für Eigenvorsorge verstärkt. Die Privatisierung der Alterssicherung zielt auf den Rückzug des Staates aus der Verantwortung, sie fordert den Ausbau privater Säulen und die individuelle Eigenverantwortung, dies bedingt eine Verlagerung von Risiken vom Kollektiv zum Individuum. Im Folgenden soll herausgearbeitet werden, dass die neue Mehrsäulenarchitektur Deutschlands durchaus auf wackligen Pfeilern steht, da sie nur unzureichend in Bezug auf Teilhabechancen und Einkommensrisiken ausbalanciert ist. Nicht jeder wird vom Umbau der Alterssicherung profitieren: einerseits ergeben sich Lücken in der sozialen Absicherung und anderseits werden Einkommensungleichheiten im Alter eher verstärkt.

Wenn finanzielle Nachhaltigkeit angesichts demografischer Alterung als Begründung für den Umbau staatlicher Rentenumlage diente, so stellen sich aber auch offene Fragen in Bezug auf den eingeschlagenen Weg der Vermarktlichung und Privatisierung. Die Finanzmarkt- und Euroschuldenkrise haben Zweifel an der Tragfähigkeit kapitalgedeckter Altersvorsorge aufkommen lassen. In ausgebauten Mehrsäulensystemen wie z. B. in Großbritannien, Irland, den Niederlanden und der Schweiz haben die erheblichen Verluste von investiven Kapitalanlagen und die Niedrigzinspolitik sicherer Staatsanleihen zu Problemen der Unterfinanzierung von Leistungszusagen der Arbeitgeber (bzw. Tarifpartner) oder unerwarteten Renditeeinbußen bei individuellen Sparplanen geführt. Die Finanzialisierung (van der Zwan 2014), d. h. die zunehmende Abhängigkeit von Finanzmarktrationalitäten, hat mit etwas Zeitverzögerung auch Deutschland und andere Bismarck'sche Rentensysteme in Süd-, Zentral- und Osteuropa grundlegend verändert. Die Finanzkrise hat aber nun auch die neuen Säulen dieser Nachzügler ins Wanken gebracht. So haben einige osteuropäische Länder das Rad der Privatisierung und Vermarktlichung in der Krise wieder etwas zurückgedreht (Drahokoupil / Domonkos 2012; Naczyk / Domonkos 2016). Während in den 1990er Jahren die staatliche Rente als nicht mehr "sicher" angesehen wurde, kam es nach dem Finanzmarktcrash von 2008 zur Ernüchterung über zu hohe 
Erwartungen an die private kapitalgedeckte Altersvorsorge. Auch in Deutschland steht das Mehrsäulenmodell in der gesellschaftspolitischen und wissenschaftlichen Diskussion (Backer 2004; Nullmeier 2015).

\section{Nachhaltigkeitsprobleme der Alterssicherung}

\subsection{Finanzielle Nachhaltigkeit angesichts demografischer Alterung}

Die demografische Alterung der Gesellschaft wurde erst seit den 1990er Jahren in der internationalen öffentlichen Debatte als Grund für umfassende Rentenreformen hin zu einem Mehrsäulenmodell vorgebracht (Leimgruber 2012; World Bank 1994). Die geringere Geburtenrate und längere Lebenserwartung führten zu einer steigenden Last der Versorgung der Älteren auf den Schultern immer weniger abhängig Beschäftigter. Ohne Zweifel fuhrt der demografische Wandel zu einer zunehmenden Belastung auch im vereinten Deutschland : So wird sich über die nächsten 50 Jahre der Altenquotient, d. h. das Verhältnis der älteren Bevölkerung („,65 Plus“) gegenüber der erwerbsfähigen Bevölkerung, von heute 35 Älteren (ab 65 Jahre) je 100 Personen im Erwerbsalter bis 2060 nahezu auf 65 je 100 verdoppeln (Ebbinghaus 2015). Für die deutsche Rentenversicherung ist dabei das Verhältnis von Rentenbeziehern zu Beitragszahlern ausschlaggebend: In den 1960er Jahren zahlten 19 Mio. aktiv Versicherte die Leistungen von 9 Mio. Rentnern. Heute jedoch sind es statt 2,2 Beitragszahlern pro Rentenbezieher nur noch 1,5 (bzw. 37 Mio.), die für 26 Mio. Rentenbezugskonten aufkommen (DRV 2017). Nach Prognosen wurde zukünftig das Verhältnis von Rentnern und Beitragszahler gegen eins zu eins konvergieren, wenn nicht die Altersgrenze über 65 Jahre angehoben worden wäre bzw. auch weiterhin der Kreis der Beitragszahler ausgeweitet wird.

Neben der Anhebung der Altersgrenze wurde von internationalen Organisationen auch eine Verlagerung der Finanzierung vom Umlageverfahren auf kapitalgedeckte Altersvorsorge als nachhaltige Politik empfohlen. Neben dem ins Wanken geratenen staatlichen Umlageverfahren sollten weitere private Säulen der Zusatzversorgung eingezogen werden, die einen Kapitalstock ansparen. Ein solcher Wechsel kann jedoch aus politischen Gründen nur schrittweise vollzogen werden, da ansonsten eine Generation einerseits für die gegenwärtigen Renten aufkommen und gleichzeitig für die eigene Altersvorsorge vollständig ansparen musste. Dieses Doppelzahlerproblem (Myles / Pierson 2001) gilt als Grund für das eher schrittweise Abschmelzen staatlicher Rentenanspruche und des sukzessiven Aufbaus privater kapitalgedeckter Zusatzvorsorge.

\subsection{Soziale Nachhaltigkeit: Teilhabe und Gerechtigkeit}

Die aus finanzpolitischen, wirtschaftlichen und demografischen Gründen begründeten Reformen unter der Prärogative finanzieller Nachhaltigkeit hatten lange die Frage nach sozialpolitischer Angemessenheit der Alterseinkommenssicherung aus dem Blick verloren. Altersarmut wurde durch den Ausbau der gesetzlichen Rentenversicherung in Deutschland in den Nachkriegsjahrzehnten im Vergleich zu einigen europäischen Nachbarländern mit 
der Ausnahme von Witwen, Migranten und Geringqualifizierten weitgehend zurückgedrängt (Vogel / Motel-Klingebiel 2013). Das Risiko der Altersarmut wird aber angesichts der eingeleiteten Reformen und der Flexibilisierung der

Beschäftigungsverhältnisse in der Zukunft eher wieder ansteigen. Zudem werden neue Formen der sozialen Ungleichheit individueller Risikoverteilungen mit der Vermarktlichung und Privatisierung zunehmen, Ungleichheiten im Erwerbsleben werden sich im Alter reproduzieren, aber auch konjunkturelle Schwankungen können zu Unsicherheiten bei kapitalgedeckter Altersvorsorge führen.

Folglich besteht durchaus auch ein Problem sozialer Nachhaltigkeit im Sinne von sozialer Teilhabe und Gerechtigkeit gerade auch infolge des Reformprimats finanzieller Nachhaltigkeit. Es besteht die Gefahr des Anstiegs der Altersarmut wegen des Abbaus sozialer Leistungen, aber auch wegen zunehmender Ungleichheiten durch freiwillig private Altersvorsorge und Unsicherheiten der kapitalgedeckten Renten. Nach internationalen Studien (Hinrichs / Jessoula 2012; Meyer et al. 2007) wird sich die Flexibilisierung des Arbeitsmarktes, deren Folge $u$. a. eine Zunahme diskontinuierlicher und prekärer Arbeitsverhältnisse ist, nicht nur in den Sozialversicherungssystemen aufgrund gänzlich fehlender oder ungenügender Einzahlungen negativ auswirken, sondern auch bei der kapitalgedeckten Altersvorsorge. Diese Probleme betreffen vor allem neue und zukünftige Geburtsjahrgange mit flexiblen Beschäftigungsverhältnissen, die voraussichtlich nur noch unterdurchschnittlich in die staatliche Rente und private Vorsorge einbezahlen werden bzw. können.

\subsection{Politische Nachhaltigkeit: Legitimation von Reformen}

Die aus finanziellen Nachhaltigkeitsgründen betriebenen Reformen stoßen auch aufgrund ihrer sozialen Auswirkungen auf Legitimationsprobleme; sie konnten folglich seitens der Politik auch wieder zur Disposition gestellt werden. Gerade die jüngeren Reformschritte, insbesondere die Erhöhung des Rentenalters und die Leistungskurzungen, werden von Gewerkschaften und Teilen der Bevölkerung abgelehnt (Ebbinghaus / Naumann 2018). Wie sich in Umfragen zeigt, ist Alterssicherung, neben Gesundheitsversorgung, das Sozialpolitikfeld, das die größte öffentliche Aufmerksamkeit erfahrt, und zwar nicht nur bei Befragten im Ruhestand, sondern auch bei der erwerbstätigen Bevölkerung. Ein manifester Generationenkonflikt lässt sich in der öffentlichen Meinung nicht feststellen, auch wenn Generationengerechtigkeit in der Politik kontrovers diskutiert wird. Politiker wissen um die Unpopularität der Umbaumaßnahmen, sodass eine Abstrafung bei Wahlen droht, insbesondere angesichts des signifikanten Anteils der Älteren ab 60 Jahre (40 \% aller Wahler), aber auch nachfolgender Generationen, die gerne zu bisherigen Bedingungen in den Ruhestand gehen wurden (Ebbinghaus 2015). Es gibt aber auch Anzeichen, dass die eingeschlagene Privatisierung und Vermarktlichung zu einer schleichenden Abnahme der breiten Zustimmung staatlicher Verantwortung für die Alterssicherung beiträgt (Ebbinghaus / Naumann 2018). 
Die vergangenen drei Jahrzehnte führten zum größten Paradigmenwandel in der deutschen Rentenpolitik seit der Einführung des Umlageverfahrens vor 60 Jahren. Die Reformen seit dem Mauerfall 1989 haben den Trend zur Frühverrentung umgekehrt und ab 2001 ein staatlich-privat gemischtes Mehrsäulensystem errichtet, das die Lasten der demografischen Alterung tragen und die Lucke der Lebensstandardsicherung im Alter schließen soll. Der Generationenvertrag wurde grundlegend umgeschrieben, vielfach gegen den Willen der Gewerkschaften und größerer Teile der Bevölkerung (Ebbinghaus / Naumann 2018). Die deutschen Gewerkschaften wie auch viele Erwerbstätige sind eher kritisch in Bezug auf die Erhöhung des Rentenalters und die Privatisierung der Altersvorsorge. Die Sozialpartner haben allerdings mit der betriebliche Altersvorsorge und Entgeltumwandlung auch neue Möglichkeiten tarifpolitischer Gestaltungsspielraume (Wiß 2011). Die Erfahrungen seit der Finanzmarkt- und Euroschuldenkrise der letzten Dekade zeigen jedoch einige Probleme des eingeschlagenen Wegs auf. Weder die betriebliche Altersvorsorge noch die kapitalgedeckte Riester-Rente können die zukünftigen gesetzlichen Einkommenslücken umfassend schließen, Beschäftigungslücken ausgleichen und die bisherige Lebensstandardsicherung garantieren. Bevor wir die gegenwärtigen Herausforderungen, zukünftigen Probleme und weiteren Reformschritte erörtern können, bedarf es eines Blicks auf die bisherigen Reformen.

\section{Vermarktlichung und Rückzug aus staatlicher Rentenverantwortung}

\subsection{Nachhaltige umlagefinanzierte Renten}

Neben der demografischen Alterung engen die finanzpolitischen und wirtschaftlichen Restriktionen die umlagefinanzierte Rentenversicherung des Generationenvertrages ein. Das Beitragsaufkommen wurde zunächst an den steigenden Kostendruck angepasst. Wenn noch vor 70 Jahren der Beitrag zur Rentenversicherung bei unter $6 \%$ lag, so betrug er bereits nach der 1957er Reform $14 \%$, paritätisch geteilt zwischen Arbeitgeber und Arbeitnehmer.

Angesichts des Kostendrucks durch demografischer Alterung und Frühverrentung stieg der Beitrag dann auf einen Höchststand von 20,3 \% um 1997 / 98, u. a. auch, weil die deutsche Einheit wesentlich durch die Sozialbeitrage mitfinanziert wurde (Ritter 2007). Seit den darauf folgen(S. 471) den Leistungskurzungen wurde der Beitrag mittelfristig unter $20 \%$ gehalten, zuletzt lag er dank erhöhter Beschäftigungszahlen in den letzten Jahren bei 18,7 \%. Langfristig gilt das politische Ziel, den Beitrag nicht wieder über die $20 \%$-Schwelle steigen zu lassen. Dies erfordert bei steigender Lebenserwartung jedoch einen größeren staatlichen Zuschuss, eine Erhöhung des Rentenalters oder weitere Rentenkurzungen. Lag der Bundeszuschuss in den 1960er Jahren noch bei circa $30 \%$, so sank er zunehmend und betrug weniger als $20 \%$ in den 1990er Jahren - trotz der Zusatzkosten der deutschen Einheit. Erst in den letzten zehn Jahren wurde die staatliche Finanzierung der GRV wieder erhöht und lag 2016 bei 24,7 \%, um die versicherungsfremden Leistungen zu finanzieren. Eine weitere Erhöhung wurde einen politischen Willen zur Umverteilung voraussetzen, der über das Sozialversicherungsprinzip hinausgeht. Alternativ wird über eine Ausweitung des Versichertenkreises durch eine Erwerbstätigenrentenversicherung diskutiert, die eine 
Integration der bisherigen Sondersysteme der Beamtenversorgung, freier Berufe und nichtpflichtversicherter Selbstständiger voraussetzen wurde.

\subsection{Grundsicherung gegen Altersarmut}

Die Achillessehne der Bismarck'schen Sozialversicherung ist die Mindestsicherung, da durch Abschmelzen der staatlichen Rentenleistungen immer mehr Versicherte von Armut bedroht sind. Für viele Jahrzehnte war die Sozialhilfe die einzig verbliebene Unterstützung für ältere Alleinstehende bzw. Paare mit unzureichenden Rentenansprüchen oder Migranten mit unzureichenden Beitragsjahren. Das Grundsicherungsgesetz regelt seit 2003 die Sicherung des Lebensunterhalts für jene Mitbürger und -bürgerinnen mit oder ohne Rentenbezug im Alter ab 65 Jahren (oder bei Erwerbsminderung bereits vorab), deren Einkommen unterhalb der Bedürftigkeitsgrenze liegt. Heute erhalten mehr als eine Million Menschen bedarfsgeprüfte Leistungen der Grundsicherung, davon nahezu die Hälfte wegen Erwerbsminderung, etwas mehr wegen unzureichender Altersrente, wobei Frauen eher wegen Regelalter und Männer wegen Erwerbsminderung Grundsicherungsleistungen in Anspruch nehmen. Insgesamt bezogen 2015 nur circa 3 \% der Älteren (ab 65 Jahre) eine Grundsicherung im Alter, während es bei den Beziehern von Invalidenrenten (unter 65 Jahren) um $15 \%$ sind (DRV 2017).

Die Grundsicherung ist nicht ausreichend, um das relative Armutsgefährdungsrisiko (auf dem Niveau von 60 \% des Durchschnittseinkommens) abzufedern. Nach einer BertelsmannStudie (DIW / ZEW 2017, S. 71f.) auf Grundlage des Sozio-oekonomischen Panels (SOEP) liegt der Anteil von Armutsgefährdeten zurzeit bei den ab 65-Jahrigen bei $16 \%$ und wird, so die Prognose, auf insgesamt $20 \%$ in den nächsten zehn bis 15 Jahren ansteigen, wobei bereits jetzt viel höhere Quoten in Ostdeutschland (20\%), bei Geringqualifizierten (26\%), Migranten (31\%), Langzeitarbeitslosen (38 \%) und alleinstehenden Frauen (40\%) zu verzeichnen sind. Nach Berechnungen mit GRV-Daten (Steffen 2008) musste ein Durchschnittsverdiener (bzw. bei $70 \%$ dieses Lohnniveaus) gegenwärtig 28 (bzw. 37) Jahre in die gesetzliche Rentenversicherung einbezahlt haben, um über das Bedürftigkeitsniveau zu kommen, aber in der Zukunft (2030) bereits 34 (bzw. 45) Jahre. Während in einigen BeveridgeMehrsäulensystemen wie in Dänemark oder den Niederlanden eine ausreichende Grundrente eine notwendige Armutsvermeidungshilfe ist, bedarf es in den Bismarck'schen Rentenversicherungssystemen einer Sozialrente, die bereits bei Mindestbeitragszeiten ausbezahlt wird, wie sie in Belgien und Frankreich oder einigen sud-/osteuropäischen Ländern existiert. Der Vorteil einer Sozialrente wäre, dass sie die Legitimation der Sozialversicherung starken wurde, wenn Menschen, die lange Zeit Einzahlungen geleistet haben - und sei es auf dem niedrigen Beitragsniveau der Geringverdiener - , immerhin eine armutsvermeidende Mindestrente erhalten wurden.

\subsection{Paradigmenwechsel der Alterszeitpolitik und Erwerbsminderung}

Nicht nur die demografische Entwicklung, sondern auch der Trend zur Frühverrentung hat die umlagefinanzierte Rentenversicherung bisher belastet. Mit der Deindustrialisierung und 
Massenarbeitslosigkeit seit den 1970er Jahren wurde die Frühverrentungspraxis als sozialvertragliche Entlastung des Arbeitsmarktes und zwecks personalpolitischen Strukturwandels zunächst gefordert. Zur Zeit der Rentenreform 1957 erreichten nur $45 \%$ das Regelalter für den Bezug der Altersrente von 65, viele Rentner erhielten aufgrund ihrer eingeschränkten Gesundheit vorab eine Rente wegen Erwerbsminderung (DRV 2017). Der Anteil der Regelaltersrentenbezieher sank vor der deutschen Einheit unter $25 \%$ dank früherem Berufssende für langjährig Versicherte, Sonderregelungen für Frauen, bei Arbeitslosigkeit, Schwerbehinderung oder Erwerbsminderung. Seit der Rentenreform 1992 und der darauf folgenden Einschnitte steigt der Regelalter-Austritt wieder; heute gehen um 40 \% mit 65 oder später in den Ruhestand (DRV 2017). Die Erhöhung des Regelrentenalters auf 67 galt 2006 als umstrittene Reform der Großen Koalition unter Minister Müntefering. Sie erhöhte ab 2012 das reguläre Renteneintrittsalter für Männer und Frauen stufenweise : ab dem Jahrgang 1947 bis einschließlich Jahrgang 1958 um einen Monat pro Jahrgang; und ab 2024 für die Jahrgange ab 1959 sogar um zwei Monate pro Jahrgang, bis schließlich ab 2029 für die 1964 oder später Geborenen die Rente mit 67 gilt. Die damit intendierte Verlängerung der Erwerbsphase folgt auch der europäischen Beschäftigungsstrategie und dem Reformtrend in vielen anderen europäischen Ländern.

Die Erhöhung des Regelalters und Abschaffung von Frühverrentungsmöglichkeiten bedeutet jedoch, dass die Erwerbsminderungsleistungen eine wichtigere Rolle ein nehmen, insbesondere für jene, die aus gesundheitlichen Gründen nicht bis zum erhöhten Regelalter vollzeitlich arbeiten können. Die Reform der Rente wegen Erwerbsminderung brachte fundamentale Änderungen, wie die Abschaffung der Berufsunfähigkeitsregelung für Angestellte und umfangreiche Kurzungen der Leistungen ab 2001. Die durchschnittlichen Rentenleistungen bei Erwerbsminderung liegen mindestens $10 \%$ unterhalb der Altersrenten, insbesondere für Männer, in Ostdeutschland sind diese zudem noch niedriger. Es wird nur selten privat vorgesorgt, um diese Leistungskurzungen gerade gegenüber der bisherigen Berufsunfähigkeit auszugleichen, da die besonders betroffenen Berufsgruppen wenig finanziellen Spielraum zur Eigenvorsorge haben. Die vorzeitig Erwerbsunfähigen sind somit eher von Altersarmut betroffen als andere Rentnergruppen.

\section{Privatisierung und Finanzialisierung der Altersvorsorge}

\subsection{Nachholbedarf bei der betrieblichen Altersvorsorge}

Im Gegensatz zur Mehrsäulenarchitektur des britischen, holländischen oder Schweizer Beveridge-Modells hat in Deutschland die betriebliche Altersvorsorge (bAV) als zweite Säule lange eine eher untergeordnete Rolle als freiwillige (außertarifliche) Rentenzusage deutscher Unternehmen neben der lebensstandardsichernden GRV gespielt. Seit den jüngsten GRVReformen hat sich der Kontext wie auch die bAV gewandelt. Seit der Einführung der Entgeltumwandlungsoption 2001 hat der Arbeitnehmeranteil mit Betriebsrentenanwartschaft von 13,6 (2001) auf über 17,7 Mio. (2015) zugenommen; somit sind heute $57 \%$ aller Sozialversicherten betrieblich zusatzversichert (BMAS 2016). Die 
Entgeltumwandlung wird von einem Viertel der Beschäftigten genutzt, setzt aber entsprechende Tarifvertrage voraus, die die Bedingungen, den Umfang und die Umsetzung (inkl. Arbeitgeberbeitrage) bestimmen (Blank 2015, S. 185).

Der Zugang zur betrieblichen Altersvorsorge ist sehr ungleich über die Privatwirtschaft verteilt, während im öffentlichen Dienst Sondersysteme existieren (Wiß 2011). In den größeren Betrieben der Privatwirtschaft ab 250 Mitarbeiter sind 2015 mehr als zwei Drittel betrieblich zusatzversichert, während in den mittelständischen Betrieben (50-250 Beschäftigte) nur die Hälfte der dort Arbeitenden und noch weniger Arbeitnehmerinnen und Arbeitnehmer in Kleinbetrieben abgesichert sind (BMAS 2016). Im öffentlichen Dienst besteht durch Tarifvertrag eine umfassende Verbreitung des Versorgungswerks für alle Arbeitnehmer, die kein Beamtenruhegehalt erhalten werden. Insgesamt betragen die aktiven Anwartschaftskonten der zweiten Saule (inklusive circa 5 Mio. im öffentlichen Dienst) circa 20 Mio., ein Anstieg um mindestens 5 Mio. von 2001 bis 2015 (BMAS 2016). Die WSIBetriebsratebefragung 2015 (Baumann / Blank 2016) zeigt, dass eine Mehrheit (77 \%) der Betriebe mit Mitbestimmung eine bAV anbietet und insgesamt $55 \%$ der Beschäftigten einbezogen wurden. Besonders niedrige Deckung ergibt sich jedoch im Baugewerbe, Verkehr und Handel. Viele kleinere Betriebe sehen keinen Bedarf bei ihren Arbeitnehmerinnen und Arbeitnehmern bzw. lehnen die bAV als zu kostspielig oder kompliziert ab. Diese Verbreitungsmuster verdeutlichen, dass das "Sozialpartnermodell" meist eine Tarifbindung von Unternehmen voraussetzt und dass dort Teilhabedefizite bestehen, wo die gewerkschaftliche Vertretung schwach ist.

Die Bedeutung der zweiten Säule ist jedoch für gegenwärtige Rentner in Bezug auf den Kreis der Bezieher und der Leistungen noch eingeschränkter. Während nach einer Umfrage (BMAS 2017) jeder vierte männliche altere Befragte (65+) eine betriebliche Altersvorsorge, jeder fünfte als Beamter ein Ruhegehalt und jeder fünfte als öffentlicher Bediensteter eine Zusatzleistung erhielt, ist der Anteil bei Frauen ab 65 Jahre viel geringer (nur $7 \%$ erhalten eine bAV aus der Privatwirtschaft bzw. 14 \% im öffentlichen Dienst). Zudem liegt der Anteil der Zusatzversorgung im Osten wegen geringerer Verbreitung mit unter $5 \%$ in der Privatwirtschaft und $10 \%$ im öffentlichen Dienst viel niedriger (ebd.). Auch die Leistungshohe der so erzielten Zusatzrenten (mit der Ausnahme des Beamtenruhegehalts) nimmt eine untergeordnete Rolle gegenüber der gesetzlichen Rente ein. Der Anteil der Zusatzversorgung wird angesichts des subventionierten Ausbaus und der Entgeltumwandlung in Zukunft gegenüber der staatlichen Rente eher zunehmen, aber damit werden auch die sozialen Ungleichheiten in Zugang und Leistungshohe beträchtlicher. Soziale Ausgleichsregelungen, unzureichende Dynamisierung der Rente oder Absicherung bei Arbeitsplatzwechsel sind weitere Probleme der betrieblichen Altersvorsorge.

In Deutschland spielt der Finanzkapitalismus im Vergleich zum britischen, holländischen oder Schweizer Mehrsäulensystemen noch eine untergeordnete Rolle. In der Privatwirtschaft betrugen die bAV-Deckungsmittel Ende des Jahres 2015 in allen Durchführungswegen 575 Mrd. €, wovon die Hälfte als Ruckstellungen für 7,8 Mio. Arbeitnehmer in Form von 
Direktzusagen der Arbeitgeber verbucht werden (Schwind 2017), gefolgt von

Pensionskassen (27\% der Deckungsmittel), Direktversicherungen (11\%) und Unterstutzungskassen (7\%), während die 2005 neu geschaffene Möglichkeit eines Pensionsfonds (6\%) noch marginal ist. Mit 222 Mrd. € Anlagevermögen der bAV (7 \% des Bruttosozialprodukts) liegt Deutschland im Jahr 2017 weit hinter Großbritannien (95\%), der Schweiz (128\%) oder den Niederlanden (182\%), die alle sogar die US Pension Funds (81 \%) übertreffen (OECD 2017). Die Finanzmarkt- und Euroschuldenkrise sowie die Niedrigzinspolitik der Europäischen Zentralbank haben auch Auswirkungen auf die Verbindlichkeiten (473) der Pensionszusagen der Unternehmen. Gerade in Großbritannien gibt es einen zunehmenden Trend unter den Unternehmen, Pensionszusagen eines Gehaltsanteils wegen zu hoher Verbindlichkeiten zurückzuziehen und ihren Beschäftigten nur noch beitragsbezogene kapitaldeckte Sparplane ohne Verzinsungsgarantien anzubieten (Bridgen / Meyer 2005). Auch in den Niederlanden werden zunehmend die tariflichen Rentenfonds von einer Leistungszusage auf eine gemischte Finanzierungsform umgestellt (Anderson 2017). Dies bedeutet, dass der Trend in der zweiten betrieblichen Saule hin zu einer Individualisierung der Risiken geht, wie dies bei individuellen Vorsorgemodellen (z. B. Riester-Rente) bereits der Fall ist.

\subsection{Die freiwillige Riester-Rente auf dem Prüfstand}

Die Riester-Rente wurde als freiwillige Zusatzversorgung durch die rot-grune Regierung Schroder unter Minister Riester 2001 eingeführt und durch Steuererleichterung und besondere Zulagen gefordert, die vor allem Geringverdienern, Familien und Berufsstartern unterstutzende Anreize für den Abschluss einer privaten Zusatzversicherung bieten sollen. Die Einführung war nach dem Platzen der Dotcom-Blase mit nur 2 Mio. Versicherten (2002) zunächst eher schleppend, jedoch führten einige Verbesserungen zu einem Wachstumsschub - zumindest bis zum Einsetzen der Finanzmarktkrise. Vor 2008 hatten ca. 8 von ca. 38 Mio. unmittelbar oder indirekt anspruchsberechtigten Beschäftigten einen Riester-Vertrag mit staatlicher Teilforderung (BMF 2018). Gegenwärtig (aktuellste Zahlen für 2016) sind es ca. 11 Mio. Begünstigte mit Förderzulagen (bei 17 Mio. Konten), wobei die Förderquoten für Frauen (über $40 \%$ ) nahezu doppelt so hoch sind und insbesondere von Kinderzulagen profitieren, während die Männer (ca. 20 \%) eher durch Steuerentlastungen begünstigt werden. Seit 2002 stieg der Anteil der Riester-Vertrage an den heute ca. 40 Mio. Berechtigten auf über $40 \%$, wobei ein Großteil der Geringverdiener und ein Teil der Soloselbstständigen nicht individuell vorsorgen und somit ca. ein Drittel der Werktätigen ohne jegliche private oder betriebliche Zusatzversorgung dasteht (BMAS 2017).

Die freiwillige Eigenvorsorge erfordert entsprechende finanzielle Spielraume und ökonomische Kenntnisse. Von den 2500 gegenwärtig angebotenen Produkten wählen die meisten Zulagenberechtigten eine Versicherung mit Garantieverzinsung (ca. 60 \%), gefolgt von risikoreicheren Kapitalanlagen (17\%) und Bausparvertragen (14\%), während andere Produkte eher randständig sind (BMF 2018). Über 85 \% der Zulagenbegünstigten sind gesetzlich rentenversichert, gefolgt von $6 \%$ Beamtinnen und Beamten und $5 \%$ mittelbar 
Berechtigten, während andere Gruppen wie Landwirte kaum "riestern". Der durchschnittliche Gesamtbeitrag unter Förderberechtigten betrug 2014 ca. 4 \% des Nettodurchschnittsverdienstes. Neben unzureichendem Deckungsgrad werden die geringe Verzinsung, hohen Provisionen und fehlende Transparenz von Verbraucherschutzern kritisch bewertet (Blank 2011). So zeigten diese Berechnungen, dass sich viele Anlageprodukte bei durchschnittlicher Lebenserwartung nicht lohnen, es sei denn der individuelle Rentenbezug überdauert diese tatsachlich. Angesichts der bestehenden Lücken in ihrer Verbreitung, der unzureichenden Einzahlungen und der niedrigen Verzinsung kann die private Altersvorsorge nicht wirklich die Rentenlücke schließen, die durch die Absenkung der staatlichen Renten in der Zukunft eintreten wird. 5 Anstehende Reformen Auch heute steht die Sicherung der Rente als wichtigstes von zwolf Zielen auf der (Wunsch)Liste der Bürgerinnen und Bürger (so 95 \% der Befragten in einer EMNID Umfrage 4 / 2018), noch vor Kriminalität und Mietpreisbremse; andererseits wird auch eine Steuer- und Abgabenentlastung von $78 \%$ angestrebt. Tatsachlich haben die jüngsten Koalitionsverhandlungen zwischen Union und SPD auch 2018 das Rententhema erneut aufgegriffen. Die letzte Große Koalition hatte zwei umstrittene Änderungen in der Rentenpolitik im Einklang mit dem damaligen Koalitionsvertrag verabschiedet: die Rente mit 63 (zwei Jahre früher abschlagsfreier Rentenbezug für langjährig Versicherte) und die Mutterrente (Erhöhung eines Leistungspunktes für Mutter mit vor 1992 geborenen Kindern). Der neue Koalitionsvertrag vom Februar 2018 enthalt auf Wunsch der CSU eine weitere Erhöhung um einen dritten Rentenpunkt für Mutter mit drei und mehr Kindern, die vor 1992 geboren worden sind, wobei noch offen ist, wie die jährlichen 3-4 Mrd. Mehrausgaben finanziert werden sollen. Eine Umkehrung des Rentenalters wie vorher von der SPD bevorzugt, wurde nicht wiederholt, jedoch soll die Rente bei Erwerbsminderung zukünftig hoher angesetzt werden, was mit bis zu 1,8 Mrd. berechnet wird. Ein Erfolg für die SPD ist die Anerkennung einer „doppelten Haltelinie“ für die kommenden Jahre. Sie formuliert das Ziel, die gesetzliche Rente auf heutigem Niveau von 48 \% bis 2025 abzusichern, bei Bedarf auch durch Steuermittel, sodass der Beitragssatz nicht über $20 \%$ steigen wird.

In Bezug auf die anstehenden Herausforderungen soll eine Rentenkommission einen verlässlichen Generationenvertrag bis Marz 2020 erarbeiten, der Vorschlage für die Weiterentwicklung der gesetzlichen Rentenversicherung sowie der betrieblichen und privaten Altersvorsorge ab 2025 vorlegen soll. In der Zwischenzeit sieht ein wichtiges Reformvorhaben gegen Altersarmut und zur solidarischen Unterstützung der Sozialversicherung vor, Geringverdienern mit langjähriger Tätigkeit (35 Jahre Beitrage, inkl. Erziehungs- und Pflegezeiten) eine bedarfsgeprüfte Grundrente zu ermöglichen, die $10 \%$ hoher als der Sozialhilfesatz ist. Jedoch sind die Finanzierungsmodalitäten durch Steueroder Sozialversicherung noch offen. Zudem soll es einen Härtefallfonds für Rentner in Ostdeutschland geben. Ein weiteres latentes Altersarmutsproblem ist die höhere Grundsicherungsquote bei Selbstständigen ohne zureichende Altersvorsorge. In der gegenwärtigen Legislaturperiode soll deshalb auch eine „gründerfreundlich ausgestattete“ 
und insolvenzgesicherte Altersvorsorgepflicht für Selbstständige ohne berufliche Versorgungswerke eingeführt werden.

\section{Zukünftige Herausforderungen}

Für die nächste Dekade stehen auch nach Umsetzung der geplanten Reformen der Großen Koalition weitere mittel bis langfristige Herausforderungen an, die couragiertes politisches Handeln verlangen und umfassender gesellschaftlicher Antworten bedürfen. Sicherlich werden die demografische Alterung, die internationale Wettbewerbsfähigkeit und die Eurofiskalischen Restriktionen weiterhin das Korsett schnüren, in dem es gilt, die finanzielle Nachhaltigkeit eines umlagefinanzierten staatlichen Rentensystems zu gewährleisten. Seit der Finanzmarkt- und Euroschuldenkrise wurde die Hoffnung enttäuscht, eine kapitalgedeckte Altersvorsorge könne mit hohen Renditen bei geringem Risiko die Versorgungslücke füllen. Auch wenn die Niedrigzinspolitik zu Ende gehen mag, so werden die langfristigen Renditeerwartungen geringer als erhofft bleiben.

Zahlreiche Verbraucherstudien zeigen, dass die dritte Säule, die Riester-Rente, sich als ein weniger attraktives Anlageprodukt erwiesen hat als ursprünglich gedacht; es birgt auch erhebliche Unsicherheiten und Risiken der individuellen Langlebigkeit. Zudem bedarf es der Eigeninitiative, Finanzkenntnis und Voraussicht, um für das Alter hinreichend vorzusorgen. Aus sozialpolitischer Sicht ist anzumahnen, dass überhaupt nur ein Teil der Erwerbsbevölkerung vorsorgt, um die Rentenlücke zu schließen. Und selbst in dieser Teilgruppe erfolgt die Vorsorge vielfach im Umfang nicht ausreichend oder diskontinuierlich. Auch die Reinvestition der betrieblichen Altersvorsorge als Ruckstellungen werden zunehmend von Unternehmen als Belastung gesehen, und eine Verabschiedung aus Leistungszusagen zugunsten von individuellen Kapitalrenten (wie in Großbritannien) wurde auch die zweite Säule zunehmend ihres Potenzials kollektiver Absicherung berauben, es sei denn es gelingt, flachendeckende und allgemeinverbindliche Tarifabkommen wie in den Niederlanden zu verhandeln.

Auch wenn die finanzielle Nachhaltigkeit bisher im Vordergrund von Reformbemühungen stand, bedarf es auch einer Politik sozialer Nachhaltigkeit, um Armut und Ungleichheit zu minimieren und politische Legitimation für eine sozial gerechte Alterssicherung zu erreichen. Hier sind die Herausforderungen noch vielschichtiger. Angesichts der Flexibilisierung der Beschäftigungsverhältnisse und Lebensarbeitsverlaufsmuster, der ungleichen Absicherung sozialer Gruppen durch die gesetzliche Rente wie auch privater Altersvorsorge und nicht zuletzt wegen der zunehmenden Unsicherheiten zukünftiger Rentenanspruche bedarf es einer sozialen Mindestsicherung, die über eine bedarfsgeprüfte Sozialhilfe hinausgeht. Eine Ausweitung der Sozialversicherung in eine Erwerbstätigenversicherung wurde einerseits den Kreis der Teilhabe vergrößern und auch mehr Gerechtigkeit zwischen den Berufsgruppen schaffen.

Zudem gilt es, die Mindestsicherung auszubauen. Eine Grundrente ermöglicht in BeveridgeSystemen wie den Niederlanden eine Mindestabsicherung auch für Teilzeitbeschäftigte 
sowie bei häufigen Erwerbsunterbrechungen. Die bedarfsgeprüfte Grundsicherung sollte im deutschen Rentensystem weiterhin eine residuale Auffangfunktion übernehmen für all jene, die keine ausreichenden Beitragsjahre erlangen, insbesondere Migranten der ersten Generation, um Armut im Alter zu vermeiden. Als wesentliche Absicherung sollte auch eine Mindestrente (oder „Sozialrente“) für langfristig Versicherte ausreichend über dem bedarfsgeprüften Sozialhilfeminimum liegen, damit die Legitimation der Sozialversicherung aufrechterhalten werden kann. Dabei mussten entweder die notwendigen

Versicherungsjahre von 35 Jahren für eine Sozialrente merklich gesenkt werden oder aber eine Mindestbeitragszahlung für alle im Erwerbsalter verpflichtend eingeführt werden (also auch für geringfügig Beschäftigte und Nichterwerbstätige), wie dies zum Beispiel in der Schweizer AHV-Rente der Fall ist. Dies wurde zumindest zur Armutsvermeidung beitragen und die Einzahlung in die Sozialversicherung legitimieren; ein Mindestmaß an Lebensstandsicherung wurde weiterhin zusätzliche Eigenvorsorge erfordern.

Die Altersgrenzenpolitik bedarf weiterer Entwicklung, das Auslaufen der Frühverrentungspolitik wurde (mit Ausnahme der Rente mit 63) bereits umfassend umgesetzt. Von Bedeutung ist eine langfristige, verlässliche und transparente Altersgrenze, sodass sich Individuen, Arbeitgeber und Betriebsrate darauf in der Lebens- und Personalplanung einrichten können. Eine weitere Erhöhung des Ruhestandsalters auf mehr als 67 Jahre steht auf der Tagesordnung der nächsten Rentenkommission, da die Verlängerung der durchschnittlichen Lebenserwartung voraussichtlich voranschreitet. Die internationale Reformerfahrung zeigt auch hier, dass diese Schritte möglichst stufenweise und langfristig geplant werden sollten. Desto hoher jedoch das Regelalter der staatlichen Rente, umso wichtiger sind einerseits eine ausreichende Rente wegen Erwerbsminderung im Fall gesundheitlicher Probleme und andererseits private Altersvorsorge für einen freiwilligen vorgezogenen Ruhestand, der eventuelle Abschlage (475) der staatlichen Rente kompensieren kann. Skandinavische Länder versuchen mit flexibleren Ruhestandsmodellen individuellen Präferenzen entgegenzukommen; ein klar definiertes Ruhestandsalter kann jedoch auch Orientierungshilfe bieten. In jedem Fall sind die Arbeitgeber, Gewerkschaften und Betriebsrate gefordert, altersgerechte Arbeitsplatze, Weiterbildungsoptionen und gleitende Übergänge in den Ruhestand in den Betrieben zu fordern. Die Alterssicherungspolitik bedarf der Flankierung durch eine arbeitsmarktpolitische Strategie des lebenslangen Lernens und aktiven Alterns.

Neben der staatlichen Säule mussten die betrieblichen und privaten Altersvorsorgesäulen weiterentwickelt werden, um eine umfassende, ausreichende und abgesicherte Einkommenssicherung im Alter zu erreichen. Zentral ist der Zugang zu betrieblicher bzw. privater Altersvorsorge für eine möglichst breite Teilhabe. Der Anteil der Bevölkerung mit unzureichender Zusatzvorsorge ist selbst nach über zehn Jahren Werbung für die RiesterRente und tarifpolitischen Initiativen zum Ausbau der betrieblichen Altersvorsorge nicht ausreichend. Hier konnte die positive Erfahrung Großbritanniens einen gangbaren Weg aufzeichnen. Als Reaktion auf die Abnahme betrieblicher und privater Altersvorsorge wurde 
in Großbritannien stufenweise eine Pflicht für die Arbeitgeber eingeführt, ihre Mitarbeiter alle drei Jahre erneut in die betriebliche Altersvorsorge aufzunehmen, von der sie dann wieder zurücktreten können. Verhaltensökonomen hatten diese „nudging“ (Thaler / Sunstein 2009) -Strategie entwickelt, da nur wenige der so automatisch Versicherten austreten. So wurde zwar formal Freiwilligkeit zugestanden, jedoch durch eine automatische Einschreibung, Kofinanzierung und stufenweise Ausweitung eine hohe Verbreitung erreicht. In Deutschland konnte dies ebenfalls über eine Pflichteinschreibung zur betrieblichen bzw. privaten Altersvorsorge durch den Arbeitgeber gelingen. Ein anderer Pfad ware die Allgemeinverbindlichkeit von tarifvertraglichen Renten, wie sie in den Niederlanden betrieben wurde. Die Rentenlucke kann nur dann geschlossen und einschneidende soziale Ungleichheiten konnen nur dann vermieden werden, wenn es gelingt, einen besseren Deckungsgrad und Beitragskontinuitat in der Zusatzversorgung zu erreichen. Staatliche Mindestsicherung und Erwerbstatigenversicherung sollten so durch flachendeckende Zusatzversorgung erganzt werden, um Alterssicherung in der Zukunft zu garantieren.

\section{LITERATUR}

Anderson, K. (2017) : Anpassung der Alterssicherungssysteme an das veränderte Marktumfeld - ein internationaler Vergleich am Beispiel der Staaten Schweden, Niederlande und Dänemark, in : RVAktuell 4/ 2017, S. 440 -456

Bäcker, G. (2004) : Der Ausstieg aus der Sozialversicherung - Das Beispiel Rentenversicherung, in : WSI-Mitteilungen 57 (9), S. 483 -487

Baumann, H. / Blank, F. (2016) : Die Betriebliche Altersversorgung : Verbreitung und Finanzierung, Ergebnisse der WSI-Betriebsrätebefragung 2015, Wirtschaftsund Sozialwissenschaftliches Institut der Hans-Böckler-Stiftung : WSI-Report Nr. 30, Düsseldorf

Blank, F. (2011) : Die Riester-Rente : Überblick zum Stand der Forschung und sozialpolitische Bewertung nach zehn Jahren, in : Sozialer Fortschritt 60 (6), S. 109 -115

Blank, F. (2015) : Die betriebliche Altersversorgung als Gegenstand der Sozialpolitik - Stand der Dinge und Weiterentwicklung, in : Sozialer Fortschritt 64 (8), S. 184-189

BMAS (Bundesministerium für Arbeit und Soziales)(2016) : Arbeitgeber- und Trägerbefragung zur Verbreitung der betrieblichen Altersversorgung (BAV 2015), Berlin

BMAS (2017) : Alterssicherung in Deutschland 2015 (ASID 2015), Berlin

BMF (Bundesministerium der Finanzen) (2018) : Statistische Auswertungen zur RiesterFörderung, Berlin

Bridgen, P. / Meyer, T. (2005) : When do benevolent capitalists change their mind? Explaining the retrenchment of defined-benefit pensions in Britain, in : Social Policy \& Administration 39 (7), S. 764-785 
DIW (Deutsches Institut für Wirtschaftsforschung)/ZEW (Zentrum für Europäische

Wirtschaftsforschung) (2017) : Entwicklung der Altersarmut bis 2036. Trends,

Risikogruppen und Politikszenarien, Gütersloh

Drahokoupil, J. / Domonkos, S. (2012) : Averting the funding-gap crisis : East European pension reforms since 2008, in : Global Social Policy 12 (3), S. 283 -299

DRV (Deutsche Rentenversicherung) (2017) : Rentenversicherung in Zeitreihen, Berlin

Ebbinghaus, B. (2015) : Demografische Alterung und Reformen der Alterssicherung in Europa - Probleme der ökonomischen, sozialen und politischen Nachhaltigkeit, in : Kölner Zeitschrift für Soziologie und Sozialpsychologie 67 (S1), S. 325 -348

Ebbinghaus, B. / Naumann, E. (Hrsg.) (2018) : Welfare state reforms seen from below : Comparing public attitudes and organized interests in Britain and Germany, London

Hinrichs, K. / Jessoula, M. (Hrsg.) (2012) : Labour market flexibility and pension reform. Flexible today, secure tomorrow ?, Basingstoke, UK

Leimgruber, M. (2012) : The historical roots of a diffusion process : The threepillar doctrine and European pension debates (1972-1994), in : Global Social Policy 12 (1), S. 24-44

Meyer, T. (2013) : Beveridge statt Bismarck! Europäische Lehren für die Alterssicherung von Frauen und Männern in Deutschland, Friedrich Ebert Stiftung : Studie, Bonn

Meyer, T. (2015) : Die dritte Säule in der Alterssicherung - brauchen wir eine Neubewertung nach der Finanz- und Wirtschaftskrise ?, in : Sozialer Fortschritt 64 (8), S. 189-195

Meyer, T. / Bridgen, P. / Riedmüller, B. (Hrsg.) (2007) : Private pensions versus social inclusion? Non-state provision for citizens at risk in Europe, Cheltenham

Myles, J. / Pierson, P. (2001) : The comparative political economy of pension reform, in : Pierson, P. (Hrsg.) : The new politics of the welfare state, New York, S. 305-333

Naczyk, M. / Domonkos, S. (2016) : The financial crisis and varieties of pension privatization reversals in eastern Europe, in : Governance 29 (2), S. 167-184

Nullmeier, F. (2015) : Einstürzende Neubauten - Statikprobleme im Säulenmodell der Alterssicherung, in : Sozialer Fortschritt 64 (8), S. 196-202

OECD (Organisation for Economic Co-operation and Development) (2017) : Pension funds in figures, Paris

Ritter, G. A. (2007) : Der Preis der deutschen Einheit. Die Wiedervereinigung und die Krise des Sozialstaats, München

Schwind, J. (2017) : Die Deckungsmittel der betrieblichen Altersversorgung in 2015, in :

Betriebliche Altersversorgung 2017 (04), S. 349 -361 
Steffen, J. (2008) : Grundsicherung im Alter und die «Riester»-Rente : Arbeitnehmerkammer Bremen 01 / 2008

Thaler, R. H. / Sunstein, C. R. (2009) : Nudge. Improving decisions about health, wealth, and happiness, London

Vogel, C. / Motel-Klingebiel, A. (Hrsg.) (2013) : Altern im sozialen Wandel : Die Rückkehr der Altersarmut?, Wiesbaden

Wiß, T. (2011) : Der Wandel der Alterssicherung in Deutschland. Die Rolle der Sozialpartner, Wiesbaden

World Bank (1994) : Averting the old age crisis : policies to protect the old and promote growth, Oxford

van der Zwan, N. (2014) : Making sense of financialization, in : Socio-Economic Review 12 (1), S. $99-129$

\section{AUTOR:}

Bernhard Ebbinghaus, PhD, ist Professor für Sozialpolitik und Leiter des Department of Social Policy and Intervention sowie Senior Research Fellow des Green Templeton College an der Universität Oxford. Forschungsschwerpunkte : Wohlfahrtsstaatsvergleich, Alterssicherung und Arbeitsmarktpolitik.

www.ebbinghaus.blog 